\title{
Mesocosm Testing to Address Non Occupational and Exposure Driven Risk Assessment of Advanced Materials DR. ARMAND MASION
}

\section{CNRS-CEREGE}

Presenting Author: masion@cerege.fr

The recent years have witnessed an increased interest in developing (pre)-standardized procedures and guidance documents for a harmonized determination of parameters utilized in the risk assessment of nanomaterials. This consists in a variety of exposure- and hazard measurements, including "simple" physical-chemical values (e.g. size, charge) as well as biological end-points. Usually, these are standalone tests developed independently of one another. As a result, these parameters, that often are at the node of risk assessment trees, are determined under a number of experimental conditions. Nevertheless, one might wonder to which extent the non-occupational section of the life cycle of a nanomaterial can be accounted for by a sequence of tests. Indeed in an actual environment, the fate of a nanomaterial is under the control of a multiplicity of simultaneously interacting parameters, and does not necessarily obey the dichotomic process of usual risk assessment schemes. Mesocosm testing is an interesting alternative to performing separate determination of a collection of parameters. Indeed, mesocosms provide exposure and hazard data in a single experiment. The systems are allowed (and even expected) to evolve, as opposed to strictly controlled standards. Robustness of this method for monitoring the environmental effects of nanomaterials has been demonstrated. The critical factor over which the operator has a control, is the exposure scenario (e.g. pulse vs. chronic contamination, applied dose). Here we show how guidance on how to carefully design this exposure, could translate into a standardized procedure for using mesocosm testing as risk assessment tool. 